\title{
CARACTERIZAÇÃO DOS ÓBITOS NEONATAIS POR INFECÇÃO RELACIONADA À ASSISTÊNCIA À SAÚDE EM UMA MATERNIDADE ESCOLA*
}

\author{
Clara Tavares Rangel ${ }^{1}$, Nilba Lima de Souza ${ }^{2}$, Cecília Olívia Paraguai de Oliveira ${ }^{1}$
}

\begin{abstract}
RESUMO: Estudo cujo objetivo foi caracterizar os óbitos neonatais por infecção relacionada à assistência à saúde na unidade de terapia intensiva neonatal de uma Maternidade Escola, no período de agosto de 2008 a junho de 2011. Os dados foram submetidos à estatística descritiva com frequência das variáveis, maior mortalidade foi registrada em neonatos do sexo masculino, com baixo peso ao nascer, menores índices de Apgar, nascidos de parto cesariano e com idade gestacional inferior a 37 semanas. Os fatores extrínsecos estiveram relacionados ao maior tempo de hospitalização e exposição a procedimentos invasivos. As condições de nascimento dos neonatos e as intervenções assistenciais favoreceram o surgimento da infecção e, consequentemente, os óbitos, apontando a necessidade de medidas preventivas que minimizem fatores de risco evitáveis para mortalidade neonatal como resposta positiva ao serviço e à sociedade.
\end{abstract}

PALAVRAS-CHAVE: Infecção hospitalar; Unidades de terapia intensiva neonatal; Mortalidade neonatal; Enfermagem neonatal.

\section{CHARACTERIZATION OF NEONATAL DEATHS FROM HEALTHCARE-RELATED INFECTIONS IN A MATERNITY SCHOOL}

\begin{abstract}
This study aimed to characterize the neonatal deaths from infection related to health care provided in a neonatal intensive care unit in a maternity school, in the period August 2008 - June 2011. The data was analyzed using descriptive statistics with frequency of the variables. Greater mortality was recorded in male neonates, with low birth weights, lower Apgar scores, born via caesarian section and with a gestational age of below 37 weeks. The extrinsic factors were related to greater lengths of time spent hospitalized and exposed to invasive procedures. The neonates' birth conditions and the care interventions favored the appearance of infection and, consequently, the deaths, pointing to the need for preventive steps which would minimize avoidable risk factors for neonatal mortality, as a positive response to the service and to society.

KEYWORDS: Nosocomial infection; Neonatal intensive care units; Neonatal mortality; Neonatal nursing.

\section{CARACTERIZACIÓN DE LOS ÓBITOS NEONATALES POR INFECCIÓN RELACIONADA A LA ASISTENCIA A LA SALUD EN UNA MATERNIDAD ESCUELA}

RESUMEN: Estudio cuyo objetivo fue caracterizar los óbitos neonatales por infección relacionada a la asistencia a la salud en unidad de terapia intensiva neonatal de una Maternidad Escuela, en el periodo de agosto de 2008 a junio de 2011. Los datos fueron sometidos a la estadística descriptiva con frecuencia de las variables. Mayor mortalidad fue registrada en neonatos del sexo masculino, con poco peso al nacer, menores índices de Apgar, nacidos de parto cesariano y con edad gestacional inferior a 37 semanas. Los factores externos se relacionaron al mayor tiempo de hospitalización y exposición a procedimientos invasivos. Las condiciones de nacimiento de los neonatos y las intervenciones asistenciales favoreceron el surgimiento de la infección y, consecuentemente, los óbitos, indicando la necesidad de medidas preventivas que minimizen factores de riesgo evitables para mortalidad neonatal como respuesta positiva al servicio y a la sociedad.

PALABRAS CLAVES: Infección hospitalar; Unidades de terapia intensiva neonatal; Mortalidad neonatal; Enfermería neonatal.

*Este estudo é o resultado da monografia apresentada a Universidade Federal do Rio Grande do Norte - UFRN como requisito para conclusão do Curso de Graduação em Enfermagem, 2011.

${ }^{1}$ Enfermeira. Membro do Grupo de Pesquisa Enfermagem em diferentes fases da vida.

${ }^{2}$ Enfermeira. Doutora em Ciências da Saúde. Professora do Departamento de Enfermagem da UFRN. Membro do Grupo de Pesquisa Enfermagem em diferentes fases da vida. Orientadora. 


\section{INTRODUÇÃO}

As altas taxas da mortalidade neonatal registradas no Brasil configuram-se como uma violação dos Direitos Humanos de Mulheres e Crianças e um grave problema de saúde pública, tornando-se um desafio para os serviços de saúde e sociedade. Conhecer essa realidade e verificar os fatores de risco para tal ocorrência é responsabilidade de todos os profissionais de saúde e da sociedade ${ }^{(1)}$.

Nos países desenvolvidos, as taxas de infecções relacionadas à assistência à saúde (IRAS) nas Unidades de Terapia Intensiva Neonatais (UTIN) foram de 8,4 a $26 \%^{(2)}$. No Brasil, elas variam entre 18,9 a $57,7 \%$, com maior predomínio das infecções da corrente sanguínea $^{(3-4)}$. Dessa forma, nas UTIN o desafio torna-se ainda maior, pois seus usuários necessitam de cuidados especializados e constituem uma parcela da população com o sistema imunológico imaturo, tornando-se mais suscetíveis aos microorganismos invasores, sendo vítimas de hospitalização, IRAS e, consequentemente, mortalidade neonatal $(\mathrm{MN})^{(5)}$.

No Brasil, há registros de declínios de $67 \%$ do coeficiente de mortalidade infantil (CMI) devido a maior redução na faixa-etária pós-neonatal. Porém, os níveis permanecem altos quando relacionados aos neonatos, correspondente ao período de zero a 28 dias de vida, tornando-se o principal componente para a mortalidade infantil no Brasil ${ }^{(1)}$. Realidade semelhante é encontrada no Estado do Rio Grande do Norte, no qual as taxas de mortalidade infantil têm reduzido ao longo dos anos. Dados da Secretaria de Saúde Pública do Estado revelam que no período de 2000 a 2008 houve redução neste indicador de 47/1000 para 28/1000 óbitos por nascidos vivos. A MN tem se mantido estável entre 10 e 12 óbitos/1000 nascidos vivos ${ }^{(6)}$.

Cabe salientar que a hospitalização em UTIN predispõe o recém-nascido a várias técnicas invasivas, o que favorece o desenvolvimento de infecções mais severas, responsáveis pelas altas taxas de morbidademortalidade neonatal. Isso também está relacionado à qualidade da assistência prestada ao $\mathrm{RN}$, requerendo a atenção de todos os profissionais envolvidos deste cuidado $^{(5)}$. A experiência como membro da Comissão de Controle de Infecção Hospitalar de uma Maternidade Escola possibilitou a valorização de medidas de controle das infecções, bem como a preocupação em eliminar possíveis focos e procedimentos que culminem em riscos de IRAS na UTIN, acarretando em óbitos neonatais. Com isso, houve a inquietude em investigar as causas desses óbitos com vistas a contribuir para redução do número de óbitos neonatais por causas evitáveis nessa instituição.

Parte-se do pressuposto que, para prestar assistência de qualidade e garantir menores riscos aos neonatos é necessário caracterizar essa população e detectar os fatores intrínsecos e extrínsecos que estão favorecendo o aparecimento de IRAS e óbitos neonatais. Assim, o estudo visa à caracterização dos óbitos neonatais por infecção relacionada à assistência à saúde na UTIN em uma maternidade de Natal, de acordo com os registros da Comissão de Controle de Infecção Hospitalar, no período de agosto de 2008 a junho de 2011.

\section{MÉTODO}

Trata-se de um estudo documental e retrospectivo realizado no período de agosto de 2008 a junho de 2011. A escolha do campo de pesquisa foi em virtude de ser uma maternidade de referência para gravidez de risco como nascimento de risco, de caráter público, localizada na capital do Estado do Rio Grande do Norte, com 10 leitos destinados a UTIN. Incluiu-se na pesquisa todos os prontuários de neonatos nascidos na maternidade, admitidos na UTIN, e que foram a óbito por IRAS de acordo com os registros da Comissão de Controle de Infecção Hospitalar no período de coorte correspondente ao estudo.

Os casos de IRAS neonatal foram definidos de acordo com os critérios do National Nosocomial Infections Surveillance System - NNISS como sendo aquelas ocorridas no período de zero a 28 dias de vida, sem aquisição por via transplacentária, e registro de bolsa rota inferior a 24 horas. No período do estudo houve na maternidade 11.829 nascido-vivos, dos quais 190 foram a óbito. Destes, 30 foram associados a IRAS, sendo 20 neonatos e 10 tinham idade superior a 28 dias de vida.

$\mathrm{Na}$ coleta de dados utilizou-se um formulário com três blocos de investigação. A primeira relacionada à caracterização dos neonatos. A segunda refere-se aos dados obstétricos e neonatais baseados em categorias preconizadas pela literatura, tais como: idade materna (não ideal: $<20$ anos e $>35$ anos; ideal: idade $\geq 20$ e $\leq$ 35 anos); idade gestacional (pré-termo: $\leq 37$ semanas; termo: > 37 semanas); tipo de parto (vaginal ou cesáreo); classificação quanto à ruptura das membranas amnióticas ( $\leq 72$ horas; $>72$ horas); sexo; índice de Apgar no $1^{\circ}$ e no $5^{\circ}$ minuto (baixo: $<7$; adequado: $\geq 7$ ); classificação do peso ao nascer (baixo peso: $\leq 1500 \mathrm{~g}$; 
peso superior a $1500 \mathrm{~g}$ ). O último bloco refere-se aos fatores de risco que mais favorecem as IRAS, de acordo com a literatura: tempo hospitalização na UTIN; permanência dos seguintes procedimentos: cateter venoso central (CVC) e ventilação mecânica invasiva (VMI).

Foram investigados os prontuários presentes no arquivo da instituição campo de pesquisa, adotando como critério de seleção os registros das planilhas da Comissão de Controle de Infecção Hospitalar referente aos óbitos associados a IRAS, no período selecionado. Os dados coletados foram armazenados em uma planilha eletrônica e posteriormente analisados pela estatística descritiva, utilizando o software SPSS versão 17.0.

Esse estudo teve aprovação do Comitê de Ética em Pesquisa sob o n. 563/11, com autorização formal do diretor da instituição da pesquisa, por tratar-se de um estudo retrospectivo com análise documental.

\section{RESULTADOS}

A caracterização neonatal, conforme observado na tabela 1, indica predomínio do sexo masculino, e o maior número de neonatos com peso de nascimento inferior ou igual a $1500 \mathrm{~g}$. Em relação ao índice de Apgar, os índices menores que sete no primeiro minuto de vida foram predominantes, com persistências desse quadro no quinto minuto de vida em 9 neonatos.

Tabela 1- Variáveis neonatais dos óbitos associados à infecção relacionada à assistência à saúde na maternidade escola do Rio Grande do Norte, 2011. Natal, 2011

\begin{tabular}{lcc}
\hline \multicolumn{1}{c}{ Variável } & N & \% \\
\hline Sexo & 12 & $60 \%$ \\
Masculino & 8 & $40 \%$ \\
Feminino & 20 & $100 \%$ \\
Total & & \\
Classificação do peso ao nascer & 14 & $70 \%$ \\
Peso $\leq 1500 \mathrm{~g}$ & 6 & $30 \%$ \\
Peso $>1500 \mathrm{~g}$ & 20 & $100 \%$ \\
Total & & \\
Apgar primeiro minuto & 15 & $75 \%$ \\
Baixo $<7$ & 5 & $25 \%$ \\
Adequado $\geq 7$ & 20 & $100 \%$ \\
Total & & \\
Apgar quinto minuto & 9 & $45 \%$ \\
Baixo $<7$ & 11 & $55 \%$ \\
Adequado $\geq 7$ & 20 & $100 \%$ \\
Total & & \\
\hline
\end{tabular}

Os dados obstétricos demonstrados na tabela 2 revelam que a média da idade materna foi de 25 anos. A via de resolução do parto foi cirúrgica, com a idade gestacional (IG) inferior ou igual a 37 semanas e registro de 19 gestantes que tiveram bolsa rota inferior ou igual a 72 horas.

Tabela 2- Variáveis dos dados obstétricos relacionados aos óbitos neonatais associados à infecção relacionada à assistência à saúde da maternidade escola do Rio Grande do Norte. Natal, 2011

\begin{tabular}{lcc}
\hline \multicolumn{1}{c}{ Variável } & N & \% \\
\hline Idade Materna (anos) & 7 & $35 \%$ \\
Não ideal $(<20$ e $>35)$ & 13 & $65 \%$ \\
Ideal $(\geq 20$ e $\leq 35)$ & 20 & $100 \%$ \\
Total & & \\
Idade gestacional (semanas) & 17 & $85 \%$ \\
Pré-termo $\leq 37$ & 3 & $15 \%$ \\
Termo $>37$ & 20 & $100 \%$ \\
Total & & \\
Tipo de Parto & 8 & $40 \%$ \\
Vaginal & 12 & $60 \%$ \\
Cesáreo & 20 & $100 \%$ \\
Total & 19 & $95 \%$ \\
Classificação da bolsa rota (horas) \\
$\leq 72$ \\
$>72$ & 1 & $5 \%$ \\
Total & 20 & $100 \%$ \\
\hline
\end{tabular}

Dos fatores de risco elencados os maiores registros de mortalidade ocorreu nos neonatos que permaneceram hospitalizados por maior tempo na UTIN, estavam em uso de VMI e/ou CVC. Cabe destacar que 75\% dos óbitos neonatais fizeram uso concomitante de desses dispositivos invasivos. Houve o predomínio do sexo masculino em tempo de hospitalização na UTIN e no uso de VM conforme observado na Figura 1.

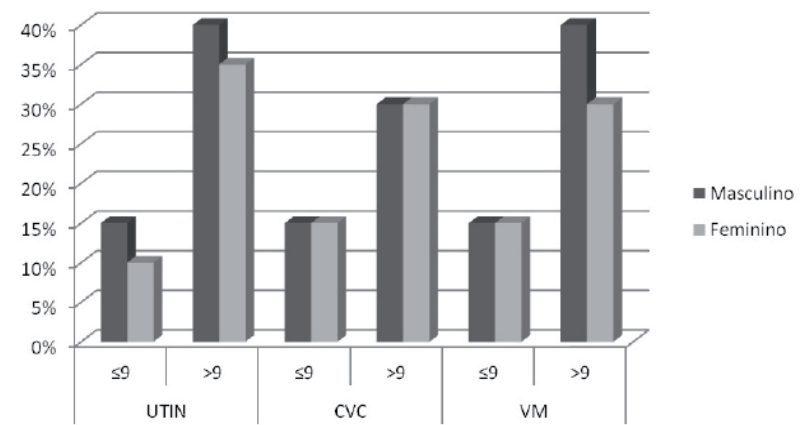

Figura 1 - Distribuição, em percentagem, dos dias de permanência na UTIN, permanência com CVC e permanência com VM, respectivamente na maternidade escola do Rio Grande do Norte. Natal, 2011 


\section{DISCUSSÃO}

A caracterização dos óbitos na UTIN investigada permitiu elucidar que a mortalidade neonatal nessa unidade de referencia estadual é representativa e requer intervenção de órgãos públicos, da instituição e dos profissionais que atuam nessa área para direcionarem suas ações com vistas a sua redução.

A idade materna não se caracterizou como fator de risco para este estudo. A faixa etária ideal das mães correspondeu a maior parte dos óbitos, contrariando alguns estudos que relacionam a menor sobrevida dos neonatos às mães adolescentes, evidenciando a fisiologia materna, a falta de estrutura sócio, psicológica e econômica que gera níveis de estresse elevados pelo não planejamento familiar ${ }^{(7)}$. Este resultado pode ser explicado pela unidade campo de pesquisa ser considerada referência na gravidez de alto risco, o que também justifica o maior número de partos cesarianos.

A literatura faz referência à idade gestacional inferior à 37 semanas como grande fator de risco para as infecções hospitalares e mortalidade neonatal ${ }^{(8)}$. Isto se confirma pela imaturidade imunológica do neonato que conforme alguns autores a fragilidade destes favorece os risco eminentes, agravos e sequelas ${ }^{(9)}$. Considera-se também que o tempo de hospitalização prolongado favorece as complicações neonatais, e consequentemente, mais vulnerabilidade desses neonatos a óbito ${ }^{(10)}$.

O tempo de bolsa rota foi superior a 24 horas em $10 \%$ dos neonatos, o que pode caracterizar infecção por via transplacentária, porém foram considerados casos de IRAS pelo estabelecimento do quadro infeccioso após sete dias de vida. É importante investigar a ruptura das membranas nos estudos sobre óbitos neonatais, para isolar os fatores que possam estar ligados à assistência ${ }^{(5)}$.

A prevalência de óbitos do sexo masculino concordou com estudos anteriores, mas sem causas elucidadas ${ }^{(11)}$. Porém, cabe ressaltar que o sexo feminino apresenta maior resistência em relação ao tempo de vida ${ }^{(5)}$.

A mortalidade relacionada ao baixo peso ao nascer, encontrada nessa investigação, é coerente com outros estudos que relatam elevado risco de morte em neonatos com menor idade gestacional e menor peso ao nascer ${ }^{(12)}$.

Os baixos índices de Apgar no primeiro minuto indicam menor vitalidade ao nascer e menor sobrevida. Fato que enfatiza a necessidade de medidas de suporte para atendimento imediato, com eficiência na assistência ofertada aos neonatos, tornando a mortalidade neonatal um evento evitável ${ }^{(13)}$. Em relação aos índices de Apgar no quinto minuto de vida, foram observados escores adequados na maior parte dos neonatos, no entanto não conseguiram sobreviver, $o$ que sugere que outros fatores impediram sua melhora progressiva. Acredita-se que quanto menor o escore de Apgar no quinto minuto de vida, menor é a chance de sobrevivência.

A exposição a fatores de riscos extrínsecos permitiu evidenciar que o maior número de obtidos esteve relacionado à tríade: maior tempo de permanência na UTIN, uso de CVC e VM. Os cateteres centrais são opções em UTIN pelo longo tempo de internação de acordo com a terapia proposta, entretanto, expõem os recém-nascidos ao risco de infecção hospitalar ${ }^{(3)}$. Em um estudo europeu com pacientes de uma UTI observou-se que as infecções relacionadas à corrente sanguínea representaram $12 \%$ das IRAS, sendo responsáveis por 14 a 38\% dos óbitos relacionados a essa causa ${ }^{(14)}$.

É conhecida a imaturidade pulmonar do organismo dos neonatos, principalmente prematuros, que muitas vezes carecem de suporte na assistência ventilatória, tornando a VM uma prática habitual nas UTIN ${ }^{(5)}$. Uma das maiores complicações que envolvem a prática de utilização da VM é a insuficiência respiratória aguda. E, após 48h de utilização do respirador mecânico, a pneumonia surge devido a presença dos tubos na via respiratória impedindo a ventilação fisiológica o que reduz a dinâmica de defesa natural das vias aéreas superiores e pulmonares, dificultando o reflexo da tosse e facilitando a penetração de microorganismos no trato inferior ${ }^{(15)}$.

Esta pesquisa verificou que os óbitos relacionados à IRAS apresentaram associação considerável com procedimentos invasivos, visto que todos os neonatos investigados foram submetidos a algum procedimento invasivo, especialmente VM e/ou CVC. Porém, pelo uso concomitante desses procedimentos houve dificuldades em analisar qual deles apresentou-se mais determinante. Além disso, o óbito neonatal é de causa multifatorial envolvendo inclusive condições obstétricas e perinatais.

Todas as variáveis investigadas nos remetem a perceber a vulnerabilidade dos neonatos em adquirir IRAS, e portanto, mais susceptíveis a mortalidade neonatal. Cabe aos profissionais pautarem suas ações no sentido de oferecer uma assistência de qualidade a gestante durante o pré-natal e que repercutam favoravelmente nas condições de nascimento, reduzindo a mortalidade neonatal decorrente da inadequação da assistência materno-infantil. Nesse sentido é imprescindível oferecer uma assistência de qualidade com 
insumos e recursos humanos adequados com vistas a preservar a vida dessas crianças ${ }^{(16)}$.

\section{CONCLUSÕES}

O perfil encontrado dos óbitos neonatais associados à IRAS na unidade campo de pesquisa revela neonatos em sua maioria do sexo masculino, que nasceram de parto cesáreo, com baixo peso e Apgar menor que sete no primeiro minuto de vida, o que nos remete às condições de nascimento do recém-nascido. Entretanto, os fatores de risco relacionados à intervenção assistencial e que ocasionaram o surgimento de infecção hospitalar foram predominantes, como o uso de procedimentos invasivos e o tempo de hospitalização.

Esses resultados foram importantes para o serviço no tocante ao retrato da UTIN com relação à assistência, uma vez que essas infecções, também são ocasionadas por intervenções terapêuticas. Esse estudo reflete a necessidade de investir em medidas preventivas, no sentido de minimizar fatores de risco evitáveis para mortalidade neonatal, como resposta positiva ao serviço e à sociedade.

É importante destacar que as gestantes de risco devem ser identificadas precocemente no pré-natal para que possam receber uma atenção especial durante a gestação e o parto, pois são elas que geram os recém-nascidos de risco que apresentam baixo peso e imaturidade imunológica, tornando-se vulneráveis a adquirirem infecções nos primeiros dias de vida.

\section{REFERÊNCIAS}

1. Ministério da Saúde (BR). Pacto Nacional pela redução da mortalidade materna e neonatal. Brasília. Balanço das ações. $20^{\mathrm{a}}$ ed. [Internet] 2007. [acesso em 28 jun 2012] Disponível: http://bvsms.saude.gov.br/bvs/folder/ pacto_reducao_mortalidade_materna_neonatal.pdf

2. Schwab F, Geffers C, Barwolff S, Ruden H, Gastmeier P. Reducing neonatal nosocomial bloodstream infections through participation in a national surveillance system. J. Hosp. Infect. [Internet] 2007;65(4) [acesso em 12 jul 2011]. Disponível: http://ac.els-cdn.com/S01956701070 0014X/1s2.0S019567010700014Xmain.pdf?_tid=82f5a cfdb973c3858e50c13e3d52f9b9\&acdnat $=1336503950$ f212548a8834a1e96921130a2131b966

3. Couto RC, Pedrosa TM, Tofani CP, Pedroso ER. Risks factors for nosocomial infection in a neonatal intensivecareunit. Infect. Control Hosp. Epidemiol. 2006;27(6):571-5.
4. Pinheiro MSB, Nicoletti C, Boszczowsk I, Puccini DMT, Ramos SRTS. Infecção hospitalar em Unidade de Terapia Intensiva Neonatal: há influência do local de nascimento? Rev. paul. pediatr. 2009;27(1):6-14.

5. Ribeiro IC. Os óbitos neonataiscom associação de complicações infecciosas: o enfermeiro pesquisando os riscos inerentes [tese]. Rio de Janeiro (RJ): Universidade Federal do Rio de Janeiro; 2010.

6. Mortalidade infantil está em queda no RN, diz secretaria. Correio da Tarde [Internet]. Natal e Mossoró; 2010 [acesso em 24 mar 2011]. Disponível: http://www. correiodatarde.com.br/editorias/correio_natal-56795

7. Schoeps D, Almeida MF, Alencar GP, França Jr. I, Novaes HMD, Siqueira AAF, et al. Fatores de risco para mortalidade neonatal precoce. Rev. Saúde Pública 2007;41(6):1013-22.

8. Goulart AP, Valle CF, Dal-Pizzol F, Cancelier ACL. Fatores de risco para o desenvolvimento de sepse neonatal precoce em Hospital da rede pública do Brasil. Rev. Bras. Terap. Intensiva. 2006;18(2):148-53.

9. Ramos HAC, Cuman RKN. Fatores de risco para a prematuridade: pesquisa documental. Esc. Anna Nery. 2009;13(2): 297-304.

10. Soares ES, Menezes GMS. Fatores associados a mortalidade neonatal precoce: análise de situação no nível local. Epidemiol. Serv. Saude. 2010;19(1)51-60.

11. Carvalho AB, Brito AS, Matsuo T. Assistência à saúde e mortalidade de recém-nascidos de muito baixo peso. Rev. Saúde Pública. 2007;41(6)1003-12.

12. Ribeiro AM, Guimarães MJ, LIMA MC, SarinhoSW, Coutinho SB. Fatores de risco para mortalidade neonatal em crianças com baixo peso ao nascer. Rev. Saúde Pública. 2009;43(2):246-55.

13. Maran E, Uchimura TT. Mortalidade Neonatal: fatores de risco em um município no sul do Brasil. Rev. Eletr. Enf. [Internet] 2008; 10(1) [acesso em 28 jun 2012]. Disponível: http://www.fen.ufg.br/revista/v10/n1/ v10n1a03.htm

14. Bonvento M. Acessos vasculares e infecção relacionada à cateter. Rev. Bras. Terap. Intensiva. 2007;19(2):227-30 .

15. Lopes FM, Lopez MF. Impacto do sistema de aspiração traqueal aberto e fechado na incidência de pneumonia associada à ventilação mecânica: revisão de literatura. Rev. Bras. Terap. Intensiva. 2009;21(1):80-8. 
16. Moreira MDS, Gaíva MAM, Bittencourt RM. Mortalidade neonatal: características assistenciais e biológicas dos recém-nascidos e de suas mães. Cogitare enferm. 2012;17(1):113-8. 